\title{
Ciudadanía enferma: el aislamiento forzoso en la profilaxis venérea argentina de la primera mitad del siglo XX
}

Resumen: El objetivo de este artículo es problematizar el concepto de ciudadanía como un derecho universal emanado de la acción exclusiva del Estado. Para ello, se analiza el proceso de construcción social del aislamiento forzoso como parte de la profilaxis venérea argentina, como resultado de la interacción entre las formulaciones normativas y las prácticas de gubernamentalidad que incluyeron a distintas reparticiones de gobierno y a los propios dolientes. Se propone que la ciudadanía enferma fue un escenario en el que se negociaron los sentidos de pertenencia, las expectativas sociales sobre cómo debían comportarse quienes se beneficiaran de ella y la experiencia de los sujetos.

Palabras clave: ciudadanía, profilaxis venérea, aislamiento forzoso, enfermos.

\section{Sick citizenship: forced isolation in the Argentine venereal prophylaxis of the first half of the twentieth century}

Abstract: This paper aims to discuss the concept of citizenship as a universal right founded on exclusive State action. It analyzes the social construction of forced isolation as an instrument of Argentinian venereal disease prophylaxis. It argues that sick citizenship was a scenario in which the meaning of belonging, the social expectations of how its beneficiaries should behave, and the social actors' experiences were negotiated. This configuration resulted from the interaction between normative and governmentality practices that involved State agents and sick people.

Keywords: citizenship, venereal prophylaxis, forced isolation, sick people.

\section{Cidadania doente: $\mathrm{o}$ isolamento forçado na profilaxia de doenças venéreas na Argentina da primeira metade do século XX}

Resumo: Este artigo tem como objetivo discutir o conceito de cidadania como direito universal emanado da ação exclusiva do Estado. Para tanto, analisa o processo de construção social do isolamento forçado como instrumento de profilaxia de doenças venéreas na Argentina. Essa configuração resultou da interação entre as práticas normativas e de governamentalidade que envolviam distintas repartições e os próprios doentes. Argumenta-se que a cidadania doente foi um cenário em que se negociou os sentidos de pertencimento, as expectativas sociais sobre como deveriam se comportar seus beneficiários, bem como as vivências dos atores sociais.

Palavras-chave: cidadania, profilaxia venérea, isolamento forçado, doentes.

Cómo citar este artículo: Carolina Biernat, "Ciudadanía enferma: el aislamiento forzoso en la profilaxis venérea argentina de la primera mitad del siglo XX”, Trashumante. Revista Americana de Historia Socia/18 [2021]: 6-27.

DOl: 10.17533/udea.trahs.n18a01

Fecha de recepción: 10 de agosto de 2020

Fecha de aprobación: 13 de noviembre de 2020

Carolina Biernat: Doctora en Historia por la Universidad Nacional del Centro de la Provincia de Buenos Aires. Investigadora independiente del Consejo Nacional de Investigaciones Científicas y Técnicas [Conicet], con lugar de trabajo en el Centro de Estudios en Historia, Cultura y Memoria [CeHCMe]. Profesora adjunta de la Universidad de Quilmes. ORCID: 0000-0002-3636-5082.

.Correo electrónico: cbiernat@yahoo.com 


\section{Ciudadanía enferma: el aislamiento forzoso en la profilaxis venérea argentina de la primera mitad del siglo XX}

Carolina Biernat

\section{Introducción}

a denuncia, el aislamiento y el tratamiento obligatorios de las personas que Ladecen una enfermedad contagiosa no constituyen una estrategia sanitaria novedosa ni privativa de la actual circunstancia de transmisión de COVID 19. Su aplicación estuvo vinculada desde hace siglos a coyunturas epidémicas y a la persistencia de algunas enfermedades de origen bacteriano consideradas peligrosas por no conocerse su cura, tales como la lepra, la tuberculosis o las infecciones venéreas. En la Argentina de la primera mitad del siglo XX, se construyó un marco legal que organizó y legitimó este conjunto de intervenciones frente a las enfermedades contagiosas. En la base de la legislación se encontraba la maduración de un concepto de salud pública de acuerdo con el que los padecimientos transmisibles podían afectar negativamente a la reproducción cuantitativa y cualitativa de la población.

En un contexto de guerras, de crisis capitalista mundial y de reconfiguración de los Estados nacionales, los discursos poblacionistas asociaron el crecimiento del factor humano con la potencialidad de modernización económica, política y social de los países, en la medida que proveía trabajadores, consumidores, ciudadanos y soldados saludables y compatibles con una supuesta homogeneidad "racial" que sería la base de una ciudadanía exenta de conflictos. Dicha premisa reforzó la catalogación de las enfermedades transmisibles como un problema de orden público. En consecuencia, el derecho a la libertad individual, garantizado por la Constitución nacional, fue modulado por reformas legislativas que incluyeron el contagio deliberado como delito contra la salud colectiva.

Asimismo, las enfermedades de origen sexual fueron incorporadas a la catalogación de transmisibles a raíz de una serie de descubrimientos que demostraron que eran hereditarias. Su presencia en la agenda de discusión y planificación sanitaria, con fuerte protagonismo de las recomendaciones de organismos internacionales, cobró una dimensión inusitada que culminó en la sanción de la ley 12331 de 
Profilaxis de las Enfermedades Venéreas en 1936. La normativa puso el acento en los aspectos de organización sanitaria, prevención y tratamiento, y dispuso tres medidas que incorporaban a estas dolencias a la categoría de transmisibles: la obligatoriedad del tratamiento, el aislamiento de los contagiados en caso necesario y la declaración de la fuente de contagio.

La historiografia local ha avanzado en el análisis de algunas dimensiones de la profilaxis de las enfermedades venéreas inaugurada en 1936, tales como la discusión y la puesta en práctica del certificado médico prenupcial para los hombres; ${ }^{1}$ el proceso de organización de su prevención y tratamiento; ${ }^{2}$ los discursos en torno a la necesidad de la educación sexual, así como las nociones de masculinidad y feminidad que desplegaron el sector médico y los funcionarios estatales; ${ }^{3}$ la abolición de la prostitución reglamentada, ${ }^{4}$ y las formas en que la política sanitaria se interconectó con la producción y la publicidad de medicamentos. ${ }^{5}$ No obstante, la indagación de la conexión entre estas dolencias y las transmisibles, en el marco del proceso de construcción social del concepto de salud pública, no ha sido abordada aún. En atención a dicho vacío, este artículo analiza una de las disposiciones de la ley 12331: la hospitalización forzosa de todo individuo enfermo que, agotados los medios persuasivos, no se sometiera con regularidad a la cura, así como la de aquellas personas cuyo tratamiento ambulante pudiera constituir un "peligro social".

El argumento tiene como base que, a pesar de que la formulación legal de la hospitalización forzosa fue pensada como un instrumento punitivo, fundamentalmente porque se buscó aislar, incluso contra su voluntad, a quienes eran considerados un foco de contagio a fin de preservar a la población saludable, su puesta en práctica se inscribió en el contexto de la ampliación de la asistencia sanitaria como derecho social. En efecto, como lo han sugerido Diego Armus y Susana Belmartino, hacia las primeras décadas del siglo XX comenzó a madurar el concepto de salud no solo como ausencia de enfermedad, sino como condición de bienestar integral y colectivo. Dicho bienestar fue asumido como responsabilidad del Estado, el cual debía garantizarlo con legislación, políticas e infraestructura adecuadas y destinadas al conjunto de la sociedad. ${ }^{6}$ En consecuencia, la salubridad

1. Marisa A. Miranda, "Matrimonio y procreación en la ortodoxia eugénica argentina", Sociohistórica 17-18 (2005): 151-178.

2. Carolina Biernat, "Médicos, especialistas, políticos y funcionarios en la organización centralizada de la profilaxis de las enfermedades venéreas en la Argentina (1930-1954)", Anuario de Estudios Americanos 64.1 (2007): 257-288.

3. Natalia Milanesio, "Redefining Men's Sexuality, Resignifying Male Bodies: The Argentine Law of Anti-Venereal Prophylaxis, 1936", Gender \& History 17.2 (2006): 463-491; Graciela Queirolo, "Género y sexualidad en tiempos de males venéreos (Buenos Aires, 1920-1940)", Nomadías 17 (2013): 67-87.

4. Karin Grammático, "Obreras, prostitutas y mal venéreo. Un Estado en busca de la profilaxis", Historia de las mujeres en la Argentina S. XX, t. 2, coord. Fernanda Gil Lozano (Buenos Aires:Taurus, 2000).

5. Carolina Biernat y Patricio Simonetto, "Provisión pública y oferta privada de medicamentos contra las enfermedades venéreas. Argentina 1930-1945”, Revista Ciencias de la Salud 15.2 (2017): 273-291.

6. Diego Armus y Susana Belmartino, "Enfermedades, médicos y cultura higiénica", Nueva historia 
constituyó uno de los pilares fundamentales en el proceso de construcción de la política social. ${ }^{7}$ Sin embargo, asegurar el derecho a la salud de todos requería en la práctica de la negación de ciertos derechos individuales de algunas personas.

Según Rafael de la Dehesa, la ciudadanía sanitaria es uno de los mecanismos clave para decidir a quién se le concede acceso sustantivo a los derechos civiles y sociales. Se trata de aquellos individuos y comunidades que poseen un entendimiento médico moderno: conocimiento del cuerpo, la salud, la enfermedad, la práctica de la higiene y la dependencia de los médicos cuando están enfermos. Por su parte, las personas que son juzgadas como incapaces de adoptar esta moderna relación médica con el cuerpo, la enfermedad y la curación, o que se niegan a hacerlo, se convierten en sujetos antihigiénicos. ${ }^{8}$ En el caso de la hospitalización forzosa, la coartación de la libertad de aquellas personas que fueron aisladas se justificó según criterios de género, responsabilizando a las mujeres que ejercían la prostitución del contagio, y de clase, al señalar a los sectores más desvalidos de la sociedad como los más vulnerables por su dudosa moralidad y falta de cuidado higiénico.

De todos modos, si bien muchos internados contra su voluntad aceptaron pasivamente las disposiciones de las autoridades, legitimando de ese modo la estrategia sociosanitaria, algunos otros se opusieron con acciones individuales o colectivas. Esto permite considerar el problema a través del prisma conceptual de la gubernamentalidad propuesto por Michel Foucault. ${ }^{9}$ Desde esta economía específica del poder, resulta indispensable el consenso que se produce entre acciones de conocimiento y control emanadas "desde arriba" y el rol activo de los sujetos sociales en su propio autogobierno. En ese sentido, la ciudadanía sanitaria puede pensarse como un proceso de construcción social para cuyo análisis resulta indispensable ahondar en la interacción entre las formulaciones normativas y las prácticas de gubernamentalidad.

Para indagar este complejo entramado relacional, que implicó, en la cuestión de la internación forzosa, la tensión entre punitivismo y asistencia sanitaria, inclusión y exclusión, resistencia y participación, se aborda, por un lado, la discusión académica y parlamentaria que llevó a la configuración de su marco legal y, por otro, su puesta en práctica a través de un estudio de caso: el Sanatorio de Previsión Social de la ciudad de Córdoba. Como se desprende del concepto de gubernamentalidad, el análisis de la puesta en práctica de esta política no incluye solamente las acciones de las distintas reparticiones de gobierno, sino también las de los enfermos. Lejos de considerarlos como sujetos pasivos intervenidos por las

argentina. Crisis económica, avance del Estado e incertidumbre política (1930-1943), t. 7, dir. Alejandro Cattaruzza (Buenos Aires: Editorial Sudamericana, 2001) 283-329.

7. Karina Ramacciotti, "Una mirada sobre el estudio de la política social en la Argentina", Nuevo Topo 1 (2005): 123-140.

8. Rafael de la Dehesa, Incursiones queer en la esfera pública. Movimientos por los derechos sexuales en México y Brasil (México: Universidad Nacional Autónoma de México / Associação Brasileira Interdisciplinar de Aids / Sexuality Policy Watch, 2015).

9. Michel Foucault, Security, Territory, Population (Nueva York: Picador, 2009). 
instituciones médicas o por la política social, poco influyentes en la gestación y la modelación de las intervenciones públicas sanitarias, se busca profundizar en su agencia y su participación en el proceso social de construcción de la ciudadanía.

\section{El peligro de la salud pública como justificación legal}

La inclusión de la internación forzosa en la ley 12331 constituye uno de los aspectos más punitivos de la normativa. Su incorporación parece haber quedado como un remanente de la discusión parlamentaria de dos leyes que marcaron la consolidación de una política sanitaria de preservación de la sociedad considerada como saludable, por encima de los derechos individuales de aquellos que padecían alguna enfermedad infecciosa. Tanto la ley 11359, para la profilaxis de la lepra (1926), como la ley 12317, de declaración obligatoria de enfermedades transmisibles, centraron su objetivo en la preservación de la salud pública. Para ello, ambas normativas disponían la denuncia y el tratamiento obligatorios de las dolencias, el aislamiento domiciliario y, en el caso de la lepra, la reclusión en instituciones, dependiendo de la "peligrosidad" del caso.

En la década de 1920, el médico y diputado radical Leopoldo Bard presentó un conjunto de proyectos en los que postulaba que las dolencias de trasmisión sexual producían "degeneración de la raza". Con base en esta consideración de tipo eugenésico, proponía la internación obligatoria en hospitales de las personas afectadas que, por su enfermedad, representaran "peligros para la salud pública". ${ }^{10}$ En el mismo sentido, el diputado socialista Ángel Giménez, presentó en 1933 un proyecto en el que preveía la internación de las "personas de uno u otro sexo cuyos hábitos, medios de vida, vivienda" les impidieran el tratamiento ambulatorio y "cuando por el estado de contagiosidad" constituyeran "un peligro" para quienes los rodeaban. ${ }^{11}$ Pese a que se apelaba a la internación con el fin de proteger al conjunto social, en especial a las personas que tenían contacto estrecho con el enfermo, en ninguno de los dos proyectos se proponía la figura forzosa de la misma. Por otro lado, la propuesta de Giménez incluía tanto a hombres como a mujeres, lo que apuntaba a un elenco universal y desvinculaba a las prostitutas de la exclusividad del padecimiento venéreo.

En el año 1935, la Cámara Baja presentó y aprobó un nuevo proyecto en el que no se encontraba previsto el aislamiento obligatorio. Fue el Senado el que sugirió un conjunto de modificaciones e inclusiones - entre ellas la internación forzosa - redactadas por la Comisión de Legislación. Dicha Comisión estaba compuesta por tres abogados: Carlos Serrey y Laureano Landaburu, del conservador Partido Demócrata Nacional, y el socialista Alfredo Palacios. El despacho fue presentado en el Senado por Serrey, quien justificó la aparente anomalía de

10. Sesión 15 de junio de 1927. Congreso de la Nación (CN), Buenos Aires, Cámara de Diputados, $622-625$.

11. Sesión 15 de septiembre de 1933. CN, Buenos Aires, Cámara de Diputados, 411-412. 
que una "comisión integrada por abogados se permita modificar una obra legislativa realizada por médicos especialistas en la materia", arguyendo que había sido asesorada por el doctor Pedro Baliña, "que es la primera autoridad en el país en enfermedades venéreas". ${ }^{12}$ En efecto, Baliña era titular de la Cátedra de Dermatosifilología de la Facultad de Ciencias Médicas de la Universidad de Buenos Aires, había participado en las discusiones parlamentarias de la ley de profilaxis de la lepra y se constituyó como un gran referente y consultor de las autoridades sanitarias que trabajaban en el problema venéreo. Sus recomendaciones, a diferencia de las de muchos de sus colegas y de representantes de instituciones de la sociedad civil como la Liga Argentina de Profilaxis Social, que ponían el acento en los aspectos preventivos como la educación sanitaria y el certificado prenupcial, ${ }^{13}$ se centraron en estrategias más invasivas sobre la libertad individual, como la imposición de un plan de tratamiento único de la sífilis en todo el país, el registro de enfermos y la obligatoriedad de una libreta sanitaria. ${ }^{14}$

Pese a la legitimación médica utilizada por Serrey, al justificar la inclusión del artículo que disponía la hospitalización forzosa, esgrimía argumentos de tipo jurídico. Para el senador, hacía "mucho tiempo que ha dejado de aplicarse el concepto de las libertades y de los derechos absolutos", ya que "todos los derechos individuales están condicionados al interés superior de la colectividad, en este caso, en el que hay verdadero peligro". ${ }^{15}$ En ese sentido, la reforma del Código Penal de 1921 había incluido en su artículo 202 los delitos contra la salud pública (transmisión de una dolencia contagiosa) y previsto su castigo con prisión de entre tres y 15 años. A pesar de ello, las enfermedades venéreas no se encontraban mencionadas explícitamente y algunos juristas, influidos por su colega español Luis Jiménez de Azúa, quien había participado en su país en la redacción del Código Penal y criticó los alcances del argentino respecto a las dolencias de transmisión sexual, ${ }^{16}$ abogaron por una legislación que las abordara específicamente y que previera instrumentos legales punitivos para resguardar al colectivo social. Tal el caso del jurista y eugenista Carlos Bernaldo de Quirós, ${ }^{17}$ quien respaldaba el proyecto de Código Sanitario remitido por el Poder Ejecutivo al Congreso en 1934, el cual contemplaba en su articulado la asistencia y profilaxis de las enfermedades venéreas a través de dispositivos como el tratamiento obligatorio y gratuito y la libreta sanitaria individual, pero se lamentaba de que no incluyese, como en la

12. Sesión 18 de septiembre de 1936. CN, Buenos Aires, Cámara de Diputados, 269.

13. Marisa A. Miranda, "Buenos Aires, entre Eros y Tánatos. La prostitución como amenaza disgénica (1930-1955)", Dynamis 32 (2012): 93-113.

14. Pedro Baliña, "En favor de la lucha contra la sífilis en nuestro país", Revista Argentina de Dermatosifilología 14 (1930): 70-72.

15. Sesión 18 de septiembre de 1936. CN, Buenos Aires, Cámara de Diputados, 270.

16. Lucía Coppa, "El delito de contagio venéreo: enfermedad, erogeneidad y responsabilidad penal (Argentina, 1922-1936)", Revista de Historia del Derecho 58 (2019): 35-62.

17. Carlos Bernaldo de Quirós, Delincuencia venérea (Buenos Aires: s.e., 1934) 97-137. 
legislación soviética, la hospitalización forzosa y la penalización del individuo que no la cumpliese.

Las revisiones del proyecto original fueron aprobadas y pasaron a la Cámara de Diputados. En relación con la inclusión de la hospitalización forzosa, la Comisión de Higiene y Asistencia Social la aprobó sin discusión y uno de sus miembros, Tiburcio Padilla, justificó su pertinencia con el temor a que la disposición de la nueva ley para abolir el sistema reglamentado de burdeles, que los obligaba a cumplir con inspecciones sanitarias periódicas a las mujeres que trabajaban en ellos, imposibilitara el control de la salud de las prostitutas, quienes empezarían a trabajar por cuenta propia. En efecto, desde la concepción androcéntrica de la salud de la época, sostenida por académicos, hombres públicos, médicos, juristas, funcionarios sanitarios, y contenida en el proyecto de ley de profilaxis venérea, la mujer, principalmente una prostituta o alguien a quien se le atribuyera una "moral dudosa" y no fuera calificada como "decente", era siempre la portadora del mal. El hombre, por su parte, tenía la responsabilidad de cuidar su salud y la de su progenie para garantizar la reproducción cualitativa de la población, por lo que el contagio de una enfermedad de origen sexual parecía ser siempre una contingencia. Por otro lado, Padilla justificaba la intervención de las autoridades sanitarias a través de la hospitalización forzosa bajo el argumento del cuidado de la salud pública. ${ }^{18}$

La internación forzosa propuesta por el Senado fue aprobada por la Cámara de Diputados, que el 17 de diciembre la incluyó entre las disposiciones de la ley. La normativa fue reglamentada por el Poder Ejecutivo y, entre otras cosas, disponía que todos los hospitales de la nación debían destinar camas para hacer efectivo el aislamiento obligatorio. Debido a ello, se trató de una de las disposiciones más difíciles de llevar a cabo por las autoridades sanitarias. En este sentido, en 1944, los venereólogos de la ciudad de Buenos Aires se quejaban de que el recrudecimiento de los casos de sífilis ocurría "no por culpa de la ley 12331, sino, por el contrario, por su incumplimiento y por su deficiente e imperfeta aplicación”. Entre sus recomendaciones se encontraba la creación de un instituto para la internación de enfermos en periodos de máximo contagio. ${ }^{19}$

Por su parte, el jurista español Jiménez de Asúa lamentaba las acciones de los jueces en torno a la aplicación de la ley 12331. Si bien subrayó que ningún magistrado negaba el fin de salud pública de sus disposiciones, al revisar la jurisprudencia nacional, concluía que en ninguna de las sentencias de la capital en las que fueron detenidas prostitutas se hallaba "indicio alguno sobre su estado sanitario, ni sobre remisión de las enfermas a las autoridades sanitarias” y que en Tucumán solo en dos ocasiones la Cámara Criminal había ordenado la hospitalización forzosa de mujeres contagiadas. En efecto, en uno de estos casos las bailarinas de un dancing detenidas por la policía y afectadas de blenorragia habían solicitado un

18. Sesión 9 de diciembre. CN, Buenos Aires, Cámara de Diputados, 934.

19. Francisco Russo, "El problema de la profilaxis de la sífilis", Revista de la Asociación Argentina de Venerología y Profilaxis Social 7.12 (1944): 7-15. 
habeas corpus para que se dispusiera su liberación, pero la Cámara se los denegó y ordenó que fuesen trasladadas al hospital municipal porque, según los jueces, el establecimiento carcelario al que habían sido remitidas no era "el adecuado para la realización del tratamiento". ${ }^{20}$ Cabe aclarar que, pese a las críticas de Jiménez de Asúa, el Poder Judicial no estaba facultado por la ley para ordenar el aislamiento obligatorio, dado que esa prerrogativa era de las autoridades sanitarias. No obstante, la justicia intervenía en los casos en los que las mujeres apelaban a esta decisión a través de un habeas corpus y en las denuncias por contagio venéreo o por ejercer la prostitución en la vía pública. Si como resultado del proceso de las últimas dos causas las mujeres eran encontradas culpables y enviadas a prisión, eran los establecimientos carcelarios los que se ocupaban de su tratamiento médico.

En suma, si bien las disposiciones de la ley y su reglamentación eran explícitas sobre la hospitalización forzosa, su puesta en práctica fue bastante exigua. Pese al discurso de algunos especialistas médicos y juristas por posicionar a las enfermedades venéreas como un problema de salud colectiva que requería, en algunos casos, el aislamiento obligatorio, no se dispuso masivamente la puesta en marcha de instituciones destinadas para esa finalidad. De todos modos, no deben descartarse otras prácticas, como la utilización exclusiva de alguna sala de los hospitales para aquellas personas que no eran consideradas capaces de fugarse, o los dispensarios de las prisiones que atendían a la población carcelaria que padecía una dolencia de transmisión sexual. En ese sentido, el criterio de peligrosidad social como requisito de la internación forzosa era definido en relación con la capacidad de transmitir la dolencia por la exposición a la frecuencia del contacto sexual con otros, pero también por la indocilidad del paciente para aceptar el aislamiento. Como todo criterio, pese a su pretensión universal, en la práctica estaba vinculado a la interpretación de las autoridades y era atravesado por un sinnúmero de prejuicios de género y clase. Al aislar a las personas "dóciles" en pabellones especiales de los hospitales y a las "indóciles" en las cárceles, la creación de instituciones exclusivas no parecía ser una prioridad de la administración sanitaria.

\section{Aislamiento y tratamientos obligatorios como práctica sanitaria}

La internación forzosa fue un recurso legal muy poco utilizado en el territorio nacional, por lo que el caso de la provincia de Córdoba se constituyó como una de las excepciones. En 1938 fue fundado por las autoridades locales el Sanatorio de Previsión Social, donde se hizo efectivo el aislamiento de muchas mujeres con una enfermedad venérea en periodo máximo de contagio. Esta excepcionalidad puede ser enmarcada en el contexto de provincialización de las políticas sanitarias durante la década de 1930, en el que, según María José Ortiz Bergia, la salud pública fue instituida como una de las principales responsabilidades del Estado con la

20. "La Ley 12331 de Profilaxis de las Enfermedades Venéreas", Anales de Legislación Argentina (Buenos Aires) 1953: 729. 
sociedad. Dicho proceso fue el resultado de diferentes factores, como la existencia de condiciones institucionales, capacidades fiscales y administrativas, las expectativas sociales crecientes y los estímulos provenientes de las estructuras nacionales y de políticos y técnicos locales. Su puesta en marcha implicó la centralización sanitaria provincial y supuso reformas en la estructura estatal. Sus resultados más inmediatos se centraron en la expansión de los servicios de salud y en el incremento de la atención sanitaria. ${ }^{21}$

En relación con las enfermedades venéreas, su tratamiento se llevó a cabo desde las primeras décadas del siglo XX en los servicios de los hospitales, dispensarios e instituciones de encierro, tales como establecimientos para menores bajo tutela estatal o cárceles, que dependían de las autoridades municipales o provinciales, de sociedades de socorros mutuos o de organizaciones benéficas que recibían, en su mayoría, financiamiento público. Para el caso de las prostitutas, el régimen reglamentarista preveía su control sanitario periódico y el tratamiento de sus dolencias en los mismos burdeles donde trabajaban. El año 1938 fue crucial en el proceso de centralización de estos servicios. El decreto 40701 reglamentó la ley nacional 12331, creando bajo el control del Consejo Provincial de Higiene una Sección de Profilaxis de las Enfermedades Venéreas, la cual sería la encargada de dirigir y sistematizar la lucha contra las enfermedades de transmisión sexual. Por su parte, la abolición del régimen de prostíbulos patentados dejaba, conceptualmente, a las mujeres que intercambiaban sexo por dinero en igualdad de condiciones al resto de la población para recurrir a los centros de tratamiento de sus padecimientos venéreos.

Dada la decisión de la provincia de ejercer un control estricto sobre los enfermos venéreos, resultaba necesario contar con una institución apropiada para diagnosticarlos y, llegado el caso, disponer de su aislamiento forzoso. Ni los hospitales, de por sí saturados, contaban con el poder de policía para recluir a los pacientes, ni las cárceles disponían de los recursos materiales y profesionales para proceder con los tratamientos. De allí que el Sanatorio de Previsión Social fuera fundado para cumplir con estos objetivos. Sin embargo, la falta de presupuesto y de un edificio de gran capacidad determinó que las autoridades sanitarias lo destinaran exclusivamente para la internación de mujeres, en particular de prostitutas, a quienes consideraban como las principales agentes de contagio. Con todo, la nueva institución no podía ser pensada solamente, según se había propuesto, como un instrumento de control social y sanitario de los cuerpos de las prostitutas que se creía representaban una amenaza al orden. ${ }^{22}$

21. María José Ortiz Bergia, "La salud pública en transformación. La estructuración de políticas sanitarias en Córdoba-Argentina, 1930-1943", Asclepio. Revista de Historia de la Medicina y de la Ciencia 64.1 (2012): 121-146.

22. Lucía Busquier, “Trabajo sexual en Córdoba: biopolítica, sexo y cuerpos. La ley de Profilaxis de las Enfermedades Venéreas: el papel de la prensa y del Estado en la construcción de los cuerpos de las prostitutas en 1938”, Revista Interdisciplinaria de Estudios de Género de El Colegio de México 4 (2018): 1-25. 
En un nivel más simbólico, el Sanatorio fue proyectado por las autoridades del Consejo de Higiene como un dispositivo moderno de profilaxis venérea que permitiría a la provincia estar a la vanguardia del sistema sanitario nacional en la medida que pudiera solucionar un problema colectivo, pues la enfermedad se percibía como un síntoma de atraso, de barbarie y de disgenia que conspiraba contra las posibilidades de desarrollo. En ese sentido, el nombre Sanatorio de Previsión Social remitía a una institución con fines no solamente de salubridad, sino, también de promoción social, conforme a una nueva concepción de ciudadanía. Por otro lado, este recurso de un programa de modernidad periférica ${ }^{23}$ proponía a la capital como centro irradiador del fortalecimiento saludable de la raza, en la medida que preveía el diagnóstico y la eventual internación de personas provenientes de toda la provincia.

A pesar de las intenciones modernizadoras de la profilaxis venérea, el Sanatorio fue inaugurado a mediados de 1938 con muy magros recursos. En una casona antigua se dispusieron los consultorios externos para el diagnóstico y la atención de las mujeres, así como un sector de internación que contaba con 35 camas que ascendieron a 80 para 1940. El servicio estaba a cargo de un administrador, tres médicos, practicantes, enfermeras y personal de limpieza, cocina y vigilancia. ${ }^{24}$

En cuanto a su funcionamiento, la mayor parte de las mujeres internadas llegaban al hospital a instancias de la fuerza pública. A partir de la vigencia de la ley nacional 12331 en Córdoba, la policía, a través de la Sección de Moralidad Pública, tuvo un rol central en la aplicación de los aspectos punitivos de la normativa. Tal vez como rémora del periodo reglamentarista, cuando había tenido un papel privilegiado en el control de los burdeles, a partir de 1937 se abocó no solo a clausurar locales donde se ejerciera la prostitución, según lo disponía la ley de profilaxis, sino también a detener a aquellas mujeres que intercambiaban sexo por dinero en la vía pública, actividad que no estaba prohibida, a menos que se desarrollara en la calle. Además, conforme los dancings fueron ocupando el lugar de los antiguos burdeles, pero bajo un eufemismo que enmascaraba la oferta de sexo por dinero en la actividad artística de las bailarinas, la Sección de Moralidad Pública fue la encargada de inspeccionar estos establecimientos y de controlar que sus trabajadoras cumplieran periódicamente con los controles sanitarios. ${ }^{25}$ De allí que, en 1938, se realizaron una serie de allanamientos a salones de espectáculos, a fin de detener a las mujeres que trabajaban en ellos y ponerlas a disposición del Sanatorio.

Después de los primeros meses, en los que las razzias fueron protagónicas en el traslado de mujeres al Sanatorio, la mayor parte de aquellas que comenzaron a

23. Beatriz Moreyra, Cuestión social y políticas sociales en la Argentina. La modernidad periférica. Córdoba, 1900-1930 (Buenos Aires: Universidad Nacional de Quilmes, 2009) 393.

24. "Fue inaugurado el Sanatorio de Previsión Social", La Voz del Interior (Córdoba) 8 de julio de 1938: 8.

25. Noelia Rimonda, “Tecnologías de control social en defensa de la salud pública. La prostitución en la ciudad de Córdoba entre 1936 y 1954" (Trabajo de licenciatura, Universidad Nacional de Córdoba, 2012). 
llegar a sus consultorios lo hicieron voluntariamente, con el fin de someterse a los exámenes necesarios para poder trabajar en los dancings. Aunque la ley nacional y su decreto reglamentario provincial no lo ordenaban, las autoridades sanitarias, alineadas con las recomendaciones de especialistas como Pedro Baliña, dispusieron controles sanitarios periódicos a las mujeres que ejercían la prostitución. Para ello, los consultorios externos del Sanatorio recibían a quienes concurrían con una nota de Moralidad Pública. Una vez allí, se les extraía sangre para el análisis de Wasserman (diagnóstico de sífilis), el cual era remitido al laboratorio del Consejo Provincial de Higiene, y se les enviaba al Hospital Cáceres de Allende para hacerse la prueba de Mantoux (tuberculosis). Por la tarde, asistían nuevamente a los consultorios para someterse a una auscultación ginecológica. Si se comprobaba que padecían blenorragia, se comunicaba a Moralidad Pública que no se les autorizara trabajar en el dancing, y se procedía a su internación o tratamiento ambulatorio. Si no se detectaba blenorragia, debían esperar hasta obtener los resultados del laboratorio que descartaran el padecimiento de sífilis para que finalmente se les extendiera su certificado de salud. Además, toda "bailarina" debía concurrir una vez por semana en día fijo para que se le realizara un examen ginecológico y una vez por bimestre para someterse a una prueba de laboratorio. ${ }^{26}$

Según los informes del Sanatorio, fueron examinadas 2,109 mujeres entre julio de 1938 y diciembre de 1939; 776, en 1941; y 1,182, en 1942. ${ }^{27}$ En rigor, se trataba de una ínfima parte del total de los enfermos atendidos en los dispensarios, hospitales y salas de primeros auxilios cordobeses. Así, por ejemplo, según las estadísticas de la Sección de Profilaxis Venérea, en el año 1942 fueron auscultados por primera vez 11,400 enfermos venéreos (hombres y mujeres, tanto nativos como extranjeros) en el sistema sanitario provincial, de los cuales, fueron diagnosticados 3,223 con sífilis, 6,929 con blenorragia y 1,248 con otras dolencias. ${ }^{28}$

Las mujeres que padecían una dolencia venérea y que, por el grado de contagiosidad de su enfermedad o por las características de sus actividades laborales, eran consideradas peligrosas para el colectivo social eran internadas forzosamente en el Sanatorio. El tiempo de su estadía era variable y los tratamientos se basaban en lavajes cotidianos y en la aplicación de drogas bismutales y arsenicales, único tratamiento conocido en la época; el alta dependía de que el examen clínico y las pruebas de laboratorio dieran un resultado negativo. Entre 1939 y 1942 fueron internadas entre 450 y 500 enfermas al año. ${ }^{29}$ Se trataba, en su mayoría, según el director del Sanatorio, de "mujeres que realizaban la prostitución" y de "bajo nivel

26. "Informe del Dr. Luis Faya", Córdoba, 12 de julio de 1940. Archivo General de la Provincia (AGP), Córdoba, Ministerio de Gobierno, t. 45, ff. 139-143.

27. Pedro Martínez Esteves, "La aplicación de la Ley Nacional de Profilaxis Social n 12331 en el Sanatorio de Previsión Social de Córdoba”, Revista Argentina de Dermatosifilología 26.4 (1942): 776.

28. "Memoria de la Sección de Profilaxis Venérea del año 1942", Córdoba, 24 de febrero de 1943. AGP, Córdoba, Ministerio de Gobierno, t. 43, ff. 274-277.

29. "Informe del jefe de la Sección de Profilaxis Venérea", Córdoba, 15 de diciembre de 1942. AGP, Córdoba, Ministerio de Gobierno, t. 14, ff. 260-265. 
económico". Con un claro objetivo moralizante, y desde una perspectiva regeneracionista que vinculaba a la enfermedad con las condiciones sociales de las personas que la padecían, proponía que el Estado debía enseñarles “a trabajar, facilitar trabajo y educar", ya que "con un más alto estándar de vida y con el fomento de una mejor cultura, se logrará una mejor comprensión de los propósitos de la ley”. ${ }^{30}$

A pesar de presentarse como una institución modelo, el Sanatorio contó con serias limitaciones para cumplir con la cantidad de profilaxis que se proponía. En primer lugar, el número de camas era insuficiente, por lo que muchas veces las seccionales de policía y Moralidad Pública se veían obligadas a dejar sin efecto las detenciones por contravenciones a la ley 12331. El presidente del Consejo de Higiene se hacía eco de esta carencia y solicitaba con asiduidad al ministro de Gobierno la ampliación del presupuesto destinado al nosocomio. Así, por ejemplo, en 1943 requirió la incorporación de 50 camas nuevas, dado que el sanatorio se veía obligado a "dejar en libertad a las mujeres enfermas". 31

En segundo lugar, según la prensa, el edificio no reunía "ni remotamente las condiciones necesarias para un establecimiento hospitalario" y, con el correr de los años, se encontraba en un "deplorable estado". ${ }^{32}$ Las habitaciones eran "altamente antihigiénicas", faltaba ventilación adecuada y las pacientes se encontraban en una situación de "hacinamiento alarmante". ${ }^{33}$ En efecto, a pesar de que a partir de la década de 1930 la provincia emprendió la expansión de sus servicios de salud, el problema edilicio fue una demanda constante durante esos años.

En tercer lugar, los fondos destinados por la provincia al Sanatorio eran insuficientes. Según su director, en 1943 la asignación presupuestaria de 2,500 pesos mensuales para su mantenimiento resultaba exigua, pues había sido determinada en el año de inauguración del establecimiento, cuando contaba con pocas enfermas y "siendo en ese tiempo el costo de las mercaderías en general la mitad". De allí que, entre 1941 y 1943, solicitó que se le destinaran partidas especiales. Para ello, utilizó la intermediación de la presidenta y de una vocal del Consejo Arquidiocesano de la Asociación de Mujeres, ambas pertenecientes a familias patricias de la provincia que colaboraban "en la tarea de moralizar y mejorar la situación de las enfermas internadas". 34

Sin embargo, la colaboración de las entidades de beneficencia no se encontraba siempre tan alineada con las necesidades del Sanatorio. Las seculares tensiones entre las instituciones de gestión privada y pública se reeditaron con fuerza durante toda la década de 1940 y fueron una limitante para el funcionamiento

30. Martínez Esteves 775.

31. "Carta del presidente del Consejo de Higiene", Córdoba, 15 de febrero de 1943. AGP, Córdoba, Ministerio de Gobierno, t. 33, f. 115.

32. "Estado actual del Sanatorio de Previsión Social", La Voz del Interior (Córdoba) 3 de julio de 1941: 7.

33. "Preocupan las internadas", La Voz del Interior (Córdoba) 16 de marzo de 1944: 6.

34. "Nota del director del Sanatorio de Previsión Social", Córdoba, 10 de noviembre de 1943.AGP, Córdoba, Ministerio de Gobierno, t. 34, ff. 435-436. 
del nosocomio. Así, por ejemplo, ante la imposibilidad de atender las necesidades específicas de los niños que se internaban con sus madres, en 1941, el director del establecimiento solicitó al presidente del Consejo de Higiene que los llevara a alguna dependencia especializada. La repartición sanitaria provincial se dirigió a la presidenta de la Sociedad de Damas de la Providencia para que cinco menores provenientes del nosocomio fueran admitidos en la casa cuna. Ante la negativa de la asociación benéfica, el director del Consejo le propuso al ministro que se disminuyeran del presupuesto del año entrante los fondos destinados a la casa cuna, "establecimiento particular pero largamente subvencionado por la provincia, y se destinaran esos recursos al acondicionamiento de las Gotas de Leche para dar una solución definitiva y permanente a este asunto". ${ }^{35}$

En suma, el Sanatorio de Previsión Social fue pensado como una de las instituciones que formaría parte del entramado de modernización de la política sanitaria cordobesa que concebía a la salud de su población como un requisito para el desarrollo de la provincia. Los padecimientos venéreos, por su transmisibilidad a las generaciones presentes y futuras, estuvieron en el centro de las intervenciones públicas que se propusieron proteger a la salud colectiva de los focos de transmisión de las dolencias. La puesta en práctica de este objetivo consistió en el aislamiento de aquellas mujeres sobre las que se creía que, por su dudosa moral, bajos recursos y ocupación, propagarían su enfermedad al resto de la sociedad. La internación forzosa no contenía solamente un rasgo punitivo hacia una ciudadanía enferma, sino que albergaba también la intención de integrarla al colectivo al devolverle su salud y una moralidad que se creía perdida. De todos modos, el exiguo presupuesto, la precaria infraestructura y los conflictos interinstitucionales contrastaron con las expectativas de las autoridades del Consejo de Higiene de posicionar al Sanatorio como un dispositivo medular en la construcción de una nueva ciudadanía sanitaria, acorde a un programa de promoción social más general del Estado provincial.

\section{La ingratitud de los enfermos}

Si bien las expectativas de las autoridades sanitarias en relación con la nueva profilaxis venérea se vieron decepcionadas por el gobierno provincial, también lo fueron por las mujeres internadas en el Sanatorio. Ante la espera de una respuesta de aceptación sumisa y gratitud por ser curadas e integradas de esa forma a la ciudadanía sanitaria, muchas enfermas, con la complicidad de los dueños de los dancings, de amigos o de familiares, se resistieron a las disposiciones de aislamiento y tratamiento obligatorios. Es probable que se debiera a que no identificaban a las enfermedades venéreas necesariamente con un problema de salud pública y mucho menos de gravedad, pero, fundamentalmente, a que sus expectativas en torno

35. "Pedido de traslado de niños a Casa Cuna", Córdoba, marzo de 1941.AGP, Córdoba, Ministerio de Gobierno, t. 35, ff. 41-43. 
a su pertenencia al colectivo social también se veían defraudadas. En este sentido, como lo ha propuesto Marcia Ochoa, ${ }^{36}$ parte de la ciudadanía, pensada universalmente por el trazado de las políticas, devino en "ingrata" en la medida que no aceptó el pacto de gubernamentalidad, lo cuestionó y desplegó estrategias de resistencia a las disposiciones normativas. De este modo, a través de su participación, reconfiguró el trazado y los límites de pertenencia de la ciudadanía.

Los primeros en oponerse a la hospitalización forzosa fueron los dueños de las salas de baile, quienes veían en las nuevas medidas una persecución organizada en su contra por la policía y las autoridades sanitarias. Así, por ejemplo, en 1940, el dancing Florida envió a un representante para que le solicitara al director del Sanatorio que se le permitiese actuar a unas bailarinas que no habían realizado los exámenes correspondientes. Ante la negativa de la autoridad del nosocomio, Santucho Peña se dirigió directamente al presidente del Consejo de Higiene para denunciar supuestas irregularidades cometidas por el Sanatorio. Según el escrito, se les exigía a las mujeres que declararan en qué local actuaban o deseaban actuar a fin de presionar a los dueños de los establecimientos para que pagaran sobornos a cambio de la entrega de los certificados médicos. Con ese fin, según el informe, se seguía un procedimiento de diagnóstico antihigiénico que ponía en riesgo de contagio a las mujeres, se suplantaban por otras las extracciones enviadas al laboratorio y se demoraba la entrega de los resultados y el apto médico. Además, informaba que quienes eran diagnosticadas con alguna enfermedad eran internadas contra su voluntad, no se le permitía seguir su tratamiento ambulatorio y, durante su aislamiento, eran sometidas a abusivas prácticas de vigilancia. ${ }^{37}$

Ante el inicio de la investigación, el director y el administrador del Sanatorio comenzaron su defensa desprestigiando a Santucho, al confirmar que había sido despedido de un diario local por "incorrecciones" cometidas durante su labor, y a uno de los dueños del dancing, tras impugnarlo moralmente al asegurar que se había presentado en el hospital hacía un año con "sus dos nietitas de 3 y 16 meses de edad, infectadas de blenorragia". ${ }^{38}$ A través de este recurso, excluían al periodista y al dueño de la sala de la comunidad saludable y responsable que contribuía a la preservación sanitaria y moral de la población.

Si bien el argumento de defensa más fuerte fue que el nosocomio se había atenido siempre a los protocolos con las pacientes, la responsabilidad por aquellas prácticas que no habían ocurrido según lo determinado por las disposiciones se desplazó hacia las mujeres. Por ejemplo, sobre tres bailarinas a quienes se denunciaba por no haber obtenido su certificado de salud a tiempo para trabajar en el dancing, se declaró que una no se había presentado al examen médico, otra había

36. Marcia Ochoa, "La ciudadanía ingrata. Trans-latinas, participación y pertenencia en la ausencia del reconocimiento", El lugar sin límites. Revista de Estudios y Políticas de Género 1.2 (2019): 69-83.

37. "Memorial de Santucho Peña”, Córdoba, 6 de julio de 1940. AGP, Córdoba, Ministerio de Gobierno, t. 45, ff. 133-135.

38. "Informe de Luis Faya", Córdoba, 12 de julio de 1940. AGP, Córdoba, Ministerio de Gobierno, t. 45 , ff. $164-166$. 
concurrido al día siguiente de la cita y otra no había estado en ayunas al momento de presentarse y no volvió a concurrir al sanatorio. En otros casos, las enfermas que contaban con la aprobación para tratarse con un médico particular pero "bajo estricto control del hospital", no regresaron al sanatorio para su seguimiento. ${ }^{39}$ En cuanto a las internas, respondiendo a la denuncia de que por las noches el personal del hospital iluminaba con una linterna las camas, el administrador aseguró que tanto él como las enfermeras se veían obligados a hacer rondas nocturnas para descartar que se ocultasen en las camas de otra, ya que "por su naturaleza, muchas son anormales y viciosas". ${ }^{40}$ Estos casos muestran que las mujeres no eran dóciles frente a las prescripciones de la profilaxis venérea y también pone de relieve una lectura de esa indocilidad por parte de las autoridades que situaba al bajo estándar moral y la irresponsabilidad social de las féminas como génesis del contagio. En ese sentido, la enfermedad, en términos físicos y morales, las colocaba fuera del pacto social que constituía la ciudadanía sanitaria y por ello se justificaba su aislamiento.

Otro de los procedimientos utilizados para evitar la internación forzosa fue la presentación de habeas corpus. En general, eran los mismos dancings en los que trabajaban las bailarinas los que patrocinaban el recurso a través de un abogado. De esta manera, el cabaret Florida contrató al doctor Jacinto Quiroga para representar a sus empleadas. En un memorial presentado al ministro de Gobierno en 1942, el lego admitía que su estudio había presentado un sinnúmero de habeas corpus "en resguardo de la libertad individual de personas detenidas en el Sanatorio". ${ }^{41}$ Con este recurso, se ponía en cuestión el concepto de salud pública esgrimido por la ley 12331, subordinándolo a la inviolabilidad de las garantías de la libertad erigida por la Constitución nacional. A pesar de este argumento, los recursos fueron denegados por los jueces intervinientes.

En otras ocasiones, los habeas corpus eran también iniciados por algún conocido de las internas. En 1940, el amigo de una bailarina presentó un recurso alegando que había sido "privada arbitrariamente de la libertad por las autoridades del Sanatorio". Según el sujeto, la mujer no padecía enfermedad venérea alguna, no se había agotado con ella "recurso persuasivo de ninguna especie", ni era "persona ambulante que haga imposible su tratamiento, constituyéndola en un peligro social". Además, "la circunstancia de que deba ganarse la vida como bailarina de cabaret, no justifica que se cometa semejante atropello", ya que "tal medida no puede ser aplicada sin juicio previo y resolución de juez competente". ${ }^{42}$ En el escrito, no solo se apelaba al reconocimiento de la libertad

39. "Informe de Luis Faya", Córdoba, 12 de julio de 1940. AGP, Córdoba, Ministerio de Gobierno, t. 45 , ff. $160-162$.

40. "Informe de Luis Faya", Córdoba, 12 de julio de 1940. AGP, Córdoba, Ministerio de Gobierno, t. 45 , ff. $160-162$.

41. "Memorial de Jacinto Quiroga”, Córdoba, 23 de noviembre de 1942. AGP, Córdoba, Ministerio de Gobierno, t. 14, ff. 248-250.

42. Enrique Tello, “A propósito de la ley 12331”, Revista Argentina de Dermatosifilología 24.4 (1940): 578-579. 
individual de la bailarina, a quien se ponía en lugar de víctima por los prejuicios que su actividad laboral despertaba, sino que se desconocía, o se omitía conocer, que la ley 12331 facultaba a las autoridades sanitarias a disponer la hospitalización forzosa.

El juez interviniente requirió a la Jefatura de Policía y al director del Sanatorio que informaran acerca de los motivos legales que asistían a la internación de la mujer. Tanto la policía como la autoridad máxima del nosocomio ratificaron que se encontraba hospitalizada después de haberse comprobado que padecía de blenorragia. Según el director, la mujer podía constituir un "peligro social", puesto que, si se efectuase "un tratamiento ambulatorio no podría volver a su anterior ocupación" y, al no tener una entrada fija pecuniaria, era previsible que necesitara "ejercer la prostitución clandestina en más alta escala". En consecuencia, se convertiría "de mujer fusil a mujer ametralladora, en lo que refiere a la transmisión de la enfermedad".43

El juez no hizo lugar al pedido de la bailarina y, días más tarde, la mujer apeló a su decisión presentando un escrito, ahora en primera persona, pero presumiblemente asesorada por un letrado, en el que argumentaba: “¿Cómo es posible que, si yo afirmo que un funcionario me priva arbitrariamente de la libertad, clasificándome de enferma, cuando no lo estoy, basta el informe de dicho funcionario para denegarme el recurso?". Por otro lado, acusaba al juez de primera instancia de no haberle dado la oportunidad de que un médico forense probara que no estaba enferma. Por último, imputaba al director del establecimiento por prejuzgar, “que somos 'mujeres ametralladoras' en la expansión de las enfermedades venéreas". ${ }^{44}$ En sus argumentos, no solo ponía en duda el estar enferma, sino que acusaba al juez por negarle el derecho a la defensa como a todo ciudadano y al director del Sanatorio de condenarla al aislamiento no por su dolencia, sino por el ejercicio de la prostitución. Si bien estos considerandos remitían a los principios contenidos en los derechos civiles, probablemente como estrategia para establecer un diálogo con los representantes judiciales, en su escrito aparecía un elemento que podría ser considerado parte de la cultura legal popular: la resistencia a legitimar una construcción de la ciudadanía sanitaria que la excluía con base en prejuicios morales, de género y de clase.

El habeas corpus no era solo un recurso utilizado por las mujeres internadas en el Sanatorio. En 1942, Jerónimo, un paciente venéreo del Hospital de Clínicas, abandonó la internación alegando que continuaría el tratamiento con un médico particular. Por no cumplir con su compromiso, la Sección de Profilaxis intervino y solicitó al Juzgado de Instrucción que lo recluyera. El juez lo destinó a la sala de enfermería de la Cárcel de Encausados. A los pocos días, la madre de Jerónimo interpuso un habeas corpus. Según el enfermo, su internación le causaba un grave daño al ser empleado de Correos y Telégrafos y había sido advertido de 
que sería cesanteado si no se reintegraba al trabajo. Ante esta situación, y teniendo en cuenta que "el peligro de propagación de la enfermedad es menor en una persona del sexo masculino que en una mujer que ejerce el comercio sexual"y que el internado había prometido proseguir el tratamiento con un médico particular, el juez dio lugar al interdicto. ${ }^{45}$ Tras su decisión, aparecía una concepción androcéntrica de la salud para la que el padecimiento venéreo en un hombre constituía una contingencia individual, no un peligro social. Por otro lado, la condición de trabajador público del enfermo, lo ubicaba dentro de la categoría de población útil, responsable y moralmente saludable. Su palabra bastaba como garantía del tratamiento ambulatorio.

En otros casos, las mujeres internadas apelaron a otro tipo de recursos, como la fuga o el amotinamiento, para demostrar su resistencia a la decisión de ser hospitalizadas forzosamente. Una de estas fugas fue motivo de un sumario administrativo a una enfermera nocturna a la que el director del nosocomio acusó de no haber cumplido con sus tareas de vigilancia. La mujer declaró que en su ronda nocturna no había notado ninguna anomalía en las habitaciones y, por la mañana, ante la ausencia de algunas internas en una de las salas, se conformó con la explicación de dos enfermas, quienes le dijeron que sus compañeras se encontraban en el baño. La enfermera sostuvo ante el sumariante que las fugas eran frecuentes dado que no era fácil "vigilar un número de más de 70 internadas, la mayoría de las cuales tratan de todas formas de fugarse". Las razones que exponía acerca de las constantes huidas era "la clase de mujeres que aquí se internan y por la falta de comodidades de la casa para los fines para que se la destina", ${ }^{46}$

El médico, la practicante de guardia y el director coincidieron con la enfermera en sus declaraciones acerca de las causas de las reiteradas fugas. Por su parte, el sumariante hacía hincapié en que "pese a las conferencias de divulgación que realiza el director, la mayoría de las enfermas no quiere comprender los beneficios que para su salud representa su internación". ${ }^{47}$ Desde esta perspectiva, las mujeres aisladas eran catalogadas como personas desagradecidas que se resistían a los esfuerzos de la profilaxis venérea para preservar la salud del cuerpo social e integrar a mujeres enfermas a una nueva ciudadanía sanitaria.

El sumario permite rescatar, aunque de forma mediada por quien llevó a cabo la investigación, las razones por las que las mujeres se fugaron. Según una de ellas, argentina, soltera, de 30 años, tras concurrir al sanatorio para someterse al examen médico para actuar como bailarina en un dancing, se procedió a su internación por considerarla enferma. Transcurrido un mes, y al no tener éxito en su solicitud de que se le permitiera llegar a la pensión donde se alojaba para retirar su ropa y otros objetos personales, que no le habían sido remitidos porque debía una suma

45. "Informe del presidente del Consejo de Higiene", Córdoba, 12 de septiembre de 1942. AGP, Córdoba, Ministerio de Gobierno, t. 45, ff. 337-338.

46. "Sumario", Córdoba, 30 de julio de 1942. AGP, Córdoba, Ministerio de Gobierno, t. 45, ff. 437-444.

47. "Sumario", Córdoba, 30 de julio de 1942. AGP, Córdoba, Ministerio de Gobierno, t. 45, f. 450. 
de 10 pesos, planeó junto con otra compañera la posibilidad de una fuga. La otra mujer, española, de 22 años, "accidentalmente en la ciudad", declaró que, luego de un examen, fue internada por "supuesta enfermedad" y que, al pasar unas semanas, planeó con su compañera fugarse para recuperar una serie de elementos personales y ropa. ${ }^{48}$

Lo que aparece detrás de sus declaraciones es, por un lado, la no aceptación del diagnóstico de la enfermedad; ambas lo pusieron en cuestión y situaron su internamiento como una decisión unilateral en la que no había mediado su consenso. Por otro lado, las mujeres relataron su fuga como una práctica que intentaba reparar algo más importante que su salud: recuperar los pocos bienes que les quedaban en la pensión cuyo alquiler no podían seguir pagando porque se veían obstaculizadas para salir a trabajar. La privación de su libertad individual representaba para ellas algo más concreto que la suspensión de un derecho civil: era la imposibilidad de generar ingresos para sostener su vida. También significaba la pérdida de sus objetos personales, que no solo eran presentados como bienes, sino como materialidad de experiencias emocionales. En este sentido, un pañuelo, un vestido, una carta representaban recuerdos de una madre ausente, una pareja concluida o una promesa de una vida mejor. Además de las recurrentes fugas, en septiembre de 1945 se organizó un motín en el Sanatorio. La noche anterior, el médico internista había encontrado a varias enfermas durmiendo en el patio sobre colchones retirados de las salas. Por la mañana, las mujeres "continuaban en desorden" por lo que se resolvió "pedir la colaboración de la policía a fin de separar a las cabecillas del grupo". Poco después de su llegada, “el resto de las enfermas se agolpó en la puerta principal profiriendo gritos y una serie de improperios". La policía trató en vano de calmarlas y las mujeres, por el contrario, "empezaron a empujar la puerta y a despedazar los vidrios de la misma". Finalmente, "las insubordinadas fueron retiradas sin mayor resistencia", ${ }^{49}$

Según el diagnóstico del director, las mujeres reaccionaban de distinta forma frente a su internación obligatoria: una minoría acataba las indicaciones médicas y las disposiciones sobre disciplina que se les impartían, pero la mayoría, "mujeres de pésimos antecedentes, díscolas, rebeldes a toda sugestión", se resistían con manifestaciones que iban "desde la protesta verbal al daño intencional, pasando por los insultos al personal médico y a las enfermeras, por la instigación a la fuga y rebeldía y la negativa a someterse a los tratamientos". Basaban su actitud en la consideración de que eran "injustamente internadas". ${ }^{50}$ En efecto, lo que aparecía en las palabras del director era la tensión entre dos formas de entender lo que era justo. Si para la autoridad sanitaria estas mujeres eran, además de indóciles, ingratas con la asistencia que se les brindaba, las internas vivían el aislamiento obligatorio

48. "Sumario", Córdoba, 30 de julio de 1942. AGP, Córdoba, Ministerio de Gobierno, t. 45, ff. 445-446.

49. "Informe del director del Sanatorio de Previsión", Córdoba, 30 de septiembre de 1945. AGP, Córdoba, Ministerio de Gobierno, t. 36, ff. 362-364.

50. "Informe del director del Sanatorio de Previsión", Córdoba, 30 de septiembre de 1945. AGP, Córdoba, Ministerio de Gobierno, t. 36, f. 365. 
como una decisión injusta porque no se les permitía decidir por sí mismas ni seguir trabajando. La rebeldía era una forma de protesta contra lo establecido y, a su vez, un intento de participación en una ciudadanía de la que se consideraban excluidas. Una participación fuera de las normas impuestas para ello, en la que los gritos, los insultos y los golpes brotaban de unos cuerpos habituados a convivir con la violencia.

En suma, el temor de las autoridades sanitarias, policiales y carcelarias no parecía ser solo la propagación de los padecimientos venéreos, sino la expansión de la ingratitud e indocilidad de las mujeres, y de muchos dueños de las salas de bailes, para aceptar los instrumentos de profilaxis. Los actos de rebeldía eran pensados como expresión de una condición moral, e incluso psíquica y emocional, enferma que ponía en riesgo la salud de la ciudadanía. Por su parte, para muchas de las internas, la resistencia al tratamiento y al aislamiento obligatorios no eran meros actos de insumisión, sino prácticas que intentaban expresar, con formas conocidas en su vida cotidiana, su deseo de formar parte de una ciudadanía que les era negada. En la tensión entre expectativas desencontradas, acciones y demandas de reconocimiento y participación indócil, se reconfiguraron el trazado y los límites de pertenencia de la ciudadanía.

\section{Conclusiones}

Las enfermedades venéreas fueron imaginadas como enemigas de la modernización nacional, una amenaza que ponía en riesgo al orden racial, a la salud de la nación y a su capacidad de desarrollo. El aislamiento forzoso marcó una intervención estatal novedosa sobre la libertad individual y sobre aspectos hasta el momento considerados privados. Su legitimación se organizó en torno a la emergencia de un nuevo derecho: la salud pública. La construcción de una ciudadanía sanitaria —entendida como el bienestar integral y colectivo- dependió de múltiples agentes sociales que interactuaron en las formulaciones normativas y en las prácticas de gubernamentalidad.

En ese sentido, hemos analizado el instrumento sanitario de internación forzosa atendiendo a la relación entre la norma y el comportamiento; entre lo que se prescribió, lo que se implementó y lo que se acató. Si bien las disposiciones de la ley y su reglamentación eran explícitas, su puesta en práctica fue bastante exigua. En general, siguieron utilizándose para tal fin los establecimientos hospitalarios o los dispensarios de las cárceles. Aunque los postulados de la normativa se formularon atendiendo a un beneficiario universal, prevalecieron la interpretación y la discrecionalidad de las autoridades, atravesadas por preconceptos de género y clase, acerca de quiénes y por qué razones debían ser hospitalizados en contra de su voluntad. Al tratarse de una minoría de la población, la creación de instituciones exclusivas no parecía ser una prioridad de la administración sanitaria. La única excepción en todo el país la constituyó el Sanatorio de Previsión Social de Córdoba, el cual, pese a sus pretensiones de modernidad e inclusión sanitaria y 
moral, quedó atrapado en los límites presupuestarios, las interferencias interinstitucionales y las resistencias de las enfermas.

Los desajustes en la materialización de la política de aislamiento pueden ser pensados como síntoma de la colisión de las expectativas y los grados de internalización de la norma entre diversos actores que constituyeron el instrumento sanitario. De allí que reconstruimos lo que percibían como aplicable, debido o justo. Ante la expectativa de las autoridades de una respuesta de aceptación sumisa y gratitud por ser integradas a la ciudadanía sanitaria, muchas enfermas desafiaron las disposiciones de aislamiento y de tratamiento obligatorios. Algunas mujeres internadas forzosamente actuaron con "ingratitud", se negaron a aceptar el pacto de gubernamentalidad y desplegaron formas de negociación o ruptura con las disposiciones normativas. En sus argumentos, combinaron un lenguaje de derecho propio del edificio jurídico liberal, como la propiedad privada, la libertad, la movilidad o el trabajo, con otros provenientes de la cultura legal popular, como la impugnación de los prejuicios morales, de clase o de género y la defensa de la emocionalidad. La ciudadanía imaginada como enferma, siempre inacabada, insuficiente o excesiva, fue un escenario en el que se negociaron los sentidos de pertenencia, las expectativas sociales sobre cómo debían comportarse quienes se beneficiaran de ella y las formas materiales que mediaron la mundana experiencia de los dolientes.

\section{Fuentes}

\section{Manuscritas}

Archivo General de la Provincia, Córdoba (AGP)

Ministerio de Gobierno

Congreso de la Nación, Buenos Aires (CN)

Cámara de Diputados

\section{Periódicos y revistas}

Anales de Legislación Argentina (Buenos Aires) 1953.

La Voz del Interior (Córdoba) 1938, 1941, 1944.

Revista Argentina de Dermatosifilología (Buenos Aires) 1930, 1940, 1942.

Revista de la Asociación Argentina de Venerología y Profilaxis Social (Buenos Aires) 1944.

\section{Bibliografía}

Armus, Diego y Susana Belmartino. "Enfermedades, médicos y cultura higiénica”. Nueva historia argentina. Crisis económica, avance del estado e incertidumbre política (1930-1943). Tomo 7. Dir. Alejandro Cattaruzza. Buenos Aires: Editorial Sudamericana, 2001. 
Bernaldo de Quirós, Carlos. Delincuencia venérea. Buenos Aires: s.e., 1934.

Biernat, Carolina. "Médicos, especialistas, políticos y funcionarios en la organización centralizada de la profilaxis de las enfermedades venéreas en la Argentina (1930-1954)". Anuario de Estudios Americanos 64.1 (2007): 257-288.

Biernat, Carolina y Patricio Simonetto. "Provisión pública y oferta privada de medicamentos contra las enfermedades venéreas. Argentina 19301945”. Revista Ciencias de la Salud 15.2 (2017): 273-291.

Busquier, Lucía. "Trabajo sexual en Córdoba: biopolítica, sexo y cuerpos. La Ley de Profilaxis de las Enfermedades Venéreas: el papel de la prensa y del Estado en la construcción de los cuerpos de las prostitutas en 1938”. Revista Interdisciplinaria de Estudios de Género de El Colegio de México 4 (2018): 1-25.

Coppa, Lucía. "El delito de contagio venéreo: enfermedad, erogeneidad y responsabilidad penal (Argentina, 1922-1936)". Revista de Historia del Derecho 58 (2019): 35-62.

Dehesa, Rafael de la. Incursiones queer en la esfera pública. Movimientos por los derechos sexuales en México y Brasil. México: Universidad Nacional Autónoma de México / Associação Brasileira Interdisciplinar de Aids / Sexuality Policy Watch, 2015.

Foucault, Michel. Security, Territory, Population. Nueva York: Picador, 2009.

Grammático, Karin. "Obreras, prostitutas y mal venéreo. Un Estado en busca de la profilaxis". Historia de las mujeres en la Argentina S. XX. Coord. Fernanda Gil Lozano. Buenos Aires:Taurus, 2000.

Milanesio, Natalia. "Redefining Men’s Sexuality, Resignifying Male Bodies: The Argentine Law of Anti-Venereal Prophylaxis, 1936”. Gender \& History 17.2 (2006): 463-491.

Miranda, Marisa A. "Buenos Aires, entre Eros y Tánatos. La prostitución como amenaza disgénica (1930-1955)". Dynamis 32 (2012): 93-113. ."Matrimonio y procreación en la ortodoxia eugénica argentina". Sociohistórica 17-18 (2005): 151-178.

Moreyra, Beatriz. Cuestión social y políticas sociales en la Argentina. La modernidad periférica. Córdoba, 1900-1930. Buenos Aires: Universidad Nacional de Quilmes, 2009.

Ochoa, Marcia. "La ciudadanía ingrata. Trans-latinas, participación y pertenencia en la ausencia del reconocimiento". El lugar sin límites. Revista de Estudios y Políticas de Género 1.2 (2019): 69-83.

Ortiz Bergia, María José. "La salud pública en transformación. La estructuración de políticas sanitarias en Córdoba-Argentina, 1930-1943”. Asclepio. Revista de Historia de la Medicina y de la Ciencia 64.1 (2012): 121-146.

Queirolo, Graciela. "Género y sexualidad en tiempos de males venéreos (Buenos Aires, 1920-1940)". Nomadías 17 (2013): 67-87.

Ramacciotti, Karina. "Una mirada sobre el estudio de la política social en la 
Argentina”. Nuevo Topo 1 (2005): 123-140.

Rimonda, Noelia. "Tecnologías de control social en defensa de la Salud Pública. La prostitución en la ciudad de Córdoba entre 1936 y 1954". Tesis de licenciatura, Universidad Nacional de Córdoba, 2012. 